D.O.I.: $10.3895 / \mathrm{S} 1808-04482008000100004$

\title{
SISTEMAS DE CONHECIMENTO COM O USO DE COMMONKADS E ONTOLOGIAS - UM ALINHAMENTO ENTRE NEGÓCIOS E DESENVOLVIMENTO
}

\section{KNOWLEDGE SYSTEMS USING COMMONKADS AND ONTOLOGIES - AN ALIGNEMENT BETWEEN BUSINESS AND DEVELOPMENT}

\author{
Érika Suzuki ${ }^{1}$; Vanessa Suzuki ${ }^{2}$; Aline França de Abreu ${ }^{3}$; Wilton Souza ${ }^{4}$ \\ ${ }^{1}$ Federal University of Santa Catarina - UFSC - Florianópolis - Brasil erika@egc.ufsc.br \\ ${ }^{2}$ Federal University of Santa Catarina - UFSC - Florianópolis - Brasil vanessa.suzuki@egc.ufsc.br \\ ${ }^{3}$ Federal University of Santa Catarina - UFSC - Florianópolis - Brasil aline@deps.ufsc.br \\ ${ }^{4}$ Federal University of Santa Catarina - UFSC - Florianópolis - Brasil wsouza@inf.ufsc.br
}

\begin{abstract}
Resumo
Atualmente os sistemas de conhecimento, abrangem múltiplas áreas, trazendo e integrando todos os processos de negócios de uma empresa, com a finalidade de obter benefícios e competitividade perante o mercado. Porém para o desenvolvimento desses sistemas, existem inúmeras dificuldades, entre elas está o entendimento entre analistas de negócios e desenvolvedores de software, dificultando assim a compreensão do que é para ser feito, do que efetivamente foi feito; o retrabalho entre ambas as partes para que seja possivel obter o resultado final almejado, documentação discordante, tempo gasto para a criação de documentos, criação de requisitos, entre outros. Portanto o presente trabalho propõe a utilização de ontologias como linguagem comum entre analistas de negócios e desenvolvedores de software a fim de eliminar os desentendimentos, $o$ retrabalho, facilitar o reuso, entre outros benefícios. Também propõe um alinhamento dessas ontologias com a metodologia CommonKADS (Schreiber, 2002), facilitando dessa forma a modelagem do sistema de conhecimento final.
\end{abstract}

Palavras-chave: ontologias; analistas de negócios; engenheiros de software; commonkads; sistemas de conhecimento.

\section{Introdução}

A criação de sistemas de conhecimento deve ser iniciada pelo entendimento sobre o negócio a ser desenvolvido e pelo mapeamento do conhecimento envolvido. A modelagem conceitual é uma forma de tornar compreensível e replicável (abstraindo e representando) a complexidade da realidade, de forma a facilitar o entendimento dos processos do negócio de uma empresa.

Segundo Uschold et al. (1996), ontologia é o termo usado para se referir ao entendimento compartilhado em algum domínio de interesse, o qual pode ser usado como uma infra-estrutura unificada para resolver problemas, evitando a redescoberta de resultados equivalentes. Portanto, 
uma das formas para se conseguir essa linguagem comum seria o uso de ontologias, que facilitaria o reuso do conhecimento e da comunicação entre analistas de negócio e engenheiros de software.

Desta forma, na seção 2 serão apresentados alguns conceitos para que na seção 3 os mesmo sejam inter-relacionados com a finalidade de obter benefícios no desenvolvimento de sistemas de conhecimento valorizando aspectos importantes como: reuso de informações, documentação dos processos, linguagem comum utilizada no processo do desenvolvimento de sistemas, otimização do tempo gasto nas tarefas, entre outros. Na seção 3 uma proposta de aplicação é apresentada de forma a facilitar o entendimento do funcionamento da "linkagem" desses conceitos. E por fim na seção 4 serão apresentadas as conclusões.

\section{Conceitos}

\subsection{Modelos Conceituais}

Stewart (1998), já afirmava que o valor de um produto está no conteúdo intelectual, não físico. É o conhecimento aplicado que gera capacidade de produzir resultados, ou seja, competência, o que vem ressaltar o aspecto da inserção das competências pessoais na melhoria da competitividade organizacional.

A modelagem conceitual é um ponto importante para a área de sistemas por tentar representar o conhecimento, organizar as idéias e facilitar uma visualização sobre o domínio do problema. Segundo Silva (1999), com o modelo conceitual tenta-se diminuir as diferenças existentes entre o modelo mental e os modelos de representação computacional do conhecimento, permitindo que este navegue por todas as fases do ciclo de vida de um sistema, com pequena ou nenhuma perda de uma fase para outra. Assim como, força a organização a avaliar e validar as idéias entre as pessoas envolvidas, fazendo com que o Modelo Conceitual auxilie na elaboração de raciocínios, proporcionando aprendizagem sobre o sistema e quanto maior a aprendizagem melhor será a manipulação do conhecimento adquirido.

Ainda segundo Silva (1999), as principais características do Modelo Conceitual são:

- Simplicidade de compreensão pelo usuário do sistema e pelos projetistas;

- Indicação clara dos objetivos ou propósitos;

- Facilidade de controle, manipulação e comunicação dos resultados;

- Completude, no nível de abrangência necessária;

- Adaptativo, com procedimentos fáceis de modificação e atualização;

- Evolucionário, podendo iniciar-se simples tornando-se mais complexo em função da utilização. 
Existem diversas definições sobre o que são mapas conceituais. Segundo Moreira \& Buchweits (1993) mapas conceituais são diagramas que procuram destacar, esquematicamente, as relações significativas de um corpo de conhecimentos ou parte deles. Suctcliffe (1995) afirma que "...Modelos conceituais existem em diferentes manifestações. São expressões lingüísticas superficiais e em uma linguagem interna que, embora baseada na lingüística, representa uma abstração futura. Modelos conceituais são uma espécie de linguagem mental interna que representa valores reais sobre objetos e suas relações. A forma dos modelos mentais diferem entre pessoas e depende de estilos cognitivos pessoais."

\subsection{Metodologia CommonKADS}

A metodologia CommonKADS (Schreiber, 2002) foi criada para colocar "luz" no caminho do desenvolvimento e, mais importante, no porquê do projeto e implementação de um sistema de conhecimento. Isto porque, muitas vezes, uma organização necessita da automatização ou simplesmente da manutenção de conhecimentos estratégicos para sua sobrevivência no mercado competitivo em que se encontra. Entretanto um sistema modelado com o conhecimento requerido pode não atender a necessidade da organização, uma vez que sua estrutura de processos não foi levada em consideração ou, em um caso pior, nem era desconhecida por ela mesma. Sendo assim, a metodologia CommonKADS permite ao engenheiro do conhecimento mapear os processos e a estrutura da organização e, com base nessas informações, prosseguir ou não no desenvolvimento do sistema do conhecimento, este é um diferencial oferecido pela metodologia, permitindo que tal sistema tenha uma coesão maior com a organização e que o desenvolvimento seja mais claro para ambas as partes. Adicionalmente, provê ao engenheiro do conhecimento uma forma de repetir ou reusar os métodos e técnicas utilizadas no desenvolvimento de um sistema em situações futuras.

A construção de um sistema baseado em conhecimento segundo a metodologia CommonKADS tem como produto um conjunto de seis modelos que especificam todos os aspectos ligados ao software a ser desenvolvido, incluindo a organização, os recursos humanos, os aspectos de implementação e a interação entre estes. Assim, o conjunto é formado por: Modelo da Organização, Modelo da Tarefa, Modelo dos Agentes, Modelo do Projeto, Modelo de Comunicação e Modelo do Conhecimento. Os modelos são interdependentes, mas podem ser desenvolvidos em diferentes momentos do projeto e por diferentes equipes.

A metodologia CommonKADS representa uma visão da estrutura organizacional, e é composta por um conjunto de modelos, para o desenvolvimento de sistemas baseados em conhecimento. Assim, os modelos que a compõem são divididos em três grupos referentes: ao 
contexto do conhecimento, aos conceitos utilizados no domínio do conhecimento e ao artefato de conhecimento (produto final - projeto para um sistema de conhecimento).

\subsection{Ontologias}

Gruninger et al. (2002) identificaram três categorias principais no espaço de aplicações de ontologias:

(a) comunicação;

(b) inferência computacional;

(c) reutilização e organização do conhecimento.

Quanto à aplicação para comunicação, as ontologias permitem compartilhar o conhecimento e facilitar a comunicação entre as pessoas com diferentes visões e pontos de vista, sem se fixar no contexto particular de cada um. Assim, através do uso de ontologias, é possível construir um modelo normativo do sistema, permitindo identificar explicitamente as conexões entre os diferentes modelos do sistema.

Definições sem ambigüidade para os termos usados em um sistema de software foram estabelecidas, integrando diferentes perspectivas dos usuários. Pessoas de posições diferentes na organização possuem visão diferente do que a organização faz, dos objetivos e de como alcançar esses objetivos. Usando ontologias, a integração pode ser alcançada, levando as pessoas a chegarem a um acordo (Gruninger et al, 2002).

Devedzic (1999) afirma que ontologias facilitam a captura e a representação do conhecimento em níveis mais abstratos, oferecendo o esqueleto do conhecimento e uma infraestrutura para integrar bases de conhecimento no nível de conhecimento, independente de uma implementação particular

Finalmente, do ponto de vista da reutilização e organização do conhecimento, ontologias podem ser usadas para estruturar ou organizar bibliotecas ou repositórios e planejar e dominar a informação (Gruninger et al, 2002). Segundo Sowa (2000), a escolha de categorias ontológicas é o primeiro passo no desenvolvimento de qualquer sistema computacional. Não importa como serão chamadas, classes, relações, tipos ou qualquer outro nome adotado por uma subárea específica da computação. A seleção dessas categorias é que determina os limites de uma aplicação específica ou mesmo de uma família de aplicações. Qualquer distorção ou restrição embutida em nossa escolha inevitavelmente limitará a generalidade dos artefatos computacionais resultantes.

Segundo Holsapple e Joshi (2002) as ontologias têm grande importância na era das organizações baseadas em conhecimento. Entre as pessoas que adotam uma ontologia, seus termos são usados para perguntar e responder questões, fazer asserções, oferecer lembranças, descrever 
práticas e discutir investigações pertinentes à conduta do gerenciamento do conhecimento. Ainda, segundo os autores, na construção e aplicação de ontologias, é importante deixar claros dois pontos importantes: de um lado está a definição da própria ontologia, os conceitos usados no domínio e suas relações; do outro lado, estão os fatos descritos por ela. Esses fatos não fazem parte da ontologia, mas são por ela estruturados. Assim, uma ontologia deve descrever aspectos gerais, válidos para quaisquer sistemas no mesmo domínio, contendo apenas elementos essenciais. A adição de detalhes em uma ontologia a torna mais específica e, portanto, menos reutilizável. Idealmente, a ontologia deve ser mantida tão simples e ampla quanto possível.

Existem diversas definições sobre o que são ontologias e, de acordo com Gruber (1995), "uma ontologia define um domínio, ou, mais formalmente, especifica uma conceitualização acerca dele."

Uma ontologia define um vocabulário específico usado para descrever uma certa realidade, acompanhado de um conjunto de decisões explícitas, fixando de forma rigorosa o significado pretendido para o vocabulário. Uma ontologia envolve, então, um vocabulário de representação que captura os conceitos e relações em algum domínio e um conjunto de axiomas, que restringem a sua interpretação, afirma Guarino (1998).

A criação de ontologias pode ser vista como uma especificação de conhecimento e, segundo Freitas (2001), é obtido pela decomposição do conhecimento em módulos de construção com forte engajamento ontológico, ou seja, similar ao conhecimento tanto em relação à forma como ele é organizado, como com a terminologia empregada numa área específica.

De acordo com Gómez-Perez (1999), existem diferentes tipos de ontologias de acordo com seu grau de generalidade, podendo ser delineados:

- Ontologias de representação definem as primitivas de representação;

- Ontologias gerais (ou de topo) trazem definições abstratas necessárias para a compreensão de aspectos do mundo, como tempo, processos, papéis, espaço, seres, coisas, etc;

- Ontologias centrais (core ontologies) ou genéricas de domínio definem os ramos de estudo de uma área e/ou conceitos mais genéricos e abstratos desta área;

- Ontologias de domínio tratam de um domínio mais específico de uma área genérica de conhecimento, como botânica ou direito tributário;

- Ontologias de aplicação procuram solucionar um problema específico de um domínio, como identificar doenças do coração, a partir de uma ontologia de domínio de cardiologia; 
- Ontologias de tarefas descrevem tarefas de um domínio - como processos, planos, metas, escalonamentos, etc -, com uma visão mais funcional, declarativa, de um domínio;

- Ontologias de domínio propriamente ditas, tem uma visão mais epistemológica do domínio, focando nos conceitos e objetos do universo de discurso.

Encontra-se na literatura e também já disponíveis na WEB diversas ontologias, com extensões como RDF, OWL, DAML, entre outras. Estas ontologias podem ser usadas como base para a geração de outras, valendo-se da sua característica de reusabilidade. Entre as ontologias mais conhecidas estão a de Sowa (Sowa,2000) e a Cyc (Lenat \& Guba, 1990), que visava o raciocínio de senso comum.

A utilização de ontologias oferece grandes benefícios, entre eles: o seu próprio reuso e também de bases de conhecimento; a possibilidade de usuários efetuarem consultas e comparações; operações de integração e checagens de consistência através de editores de ontologias; a existência de vasta diversidade de ontologias, já prontas para o uso; facilidades de comunicação entre pessoas e agentes; possibilidade se traduzir as ontologias em diversas linguagens e formalismos de representação de conhecimento etc.

Adicionalmente, existe uma grande quantidade de editores de ontologias que facilitam a criação, consulta, transformação do formato da ontologia e até mesmo a linguagem de representação na qual será escrito o código gerado a partir da ontologia, um exemplo é o Protégé 2000 (Noy et all., 2000).

\section{Proposta de Aplicação}

Probst (2002) afirma que o mapeamento do conhecimento deve ser apoiado por uma linguagem comum que cubra os diferentes campos de conhecimento e que somente com um vocabulário controlado pode-se garantir o uso consciente de termos e classificação de informações junto as diferentes dimensões do conhecimento, e nesse contexto, as ontologias tem sido bastante utilizadas como uma forma de organizar os conceitos de um domínio e estabelecer essa linguagem comum. Por sua vez, José Palazzo (1996), que ao citar inúmeras aplicações complexas, principalmente relacionadas ao desenvolvimento de aplicações comerciais na internet, comenta: "Todas estas aplicações requerem suporte de funcionalidades avançadas e, principalmente, modelos abstratos, metodologias e ferramentas que permitam sua modelagem e projeto". Portanto com esse intuito de alinhar o negócio da empresa com os desenvolvimentos de sistemas, é proposto um processo para o desenvolvimento de sistemas de conhecimento. 
Os negócios da empresa serão analisados pelos analistas de negócios que procurarão delimitar e entender o escopo que será trabalhado, os processos a serem desenvolvidos, etc, sendo assim o analista de negócios mapeará o conhecimento desses processos de forma a já atender os modelos do CommonKADS, porém para essa proposta, para auxiliar na criação dos mapas de conhecimento e ontologias, apenas será utilizado os três primeiros modelos, uma vez que são eles os responsáveis por tentar elucidar as questões levantadas pelos autores da metodologia sobre porque o desenvolvimento de um sistema de conhecimento ajudaria nos problemas e quais possíveis soluções ele traria para a organização.

Os mapas conceituais e as ontologias poderão ser criados através dos modelos do CommonKADS, abaixo destacamos como esses modelos auxiliam a criação destes:

- O modelo da organização facilitará a identificação de problemas e oportunidades para os sistemas de conhecimento, estabelecendo viabilidade e o impacto que esse tipo de sistema causará na organização. Arenas et al (2004) ressaltam que esse modelo estrutura de forma sistemática a organização, o que, por sua vez, indica uma tentativa de esclarecer o funcionamento da organização, segundo o ponto de vista de um engenheiro do conhecimento;

- O modelo de tarefas auxiliará a destacar quais são as tarefas ou sub-processos envolvidos no processo principal do negócio da organização, através da análise de entradas e saídas, précondições, restrições e recursos e competências envolvidas nas tarefas;

- O modelo de agentes visa representar as pessoas, sistemas ou outro tipo de entidade que execute uma ou mais tarefas, descrevendo também as competências, restrições e poder dentro da organização, a metodologia também possui uma planilha para representar as características de cada agente.

As ontologias podem ser consideradas como "peça chave" para a documentação dos processos, reuso de informação, linguagem comum entre analistas de negócios e engenheiros de software entre outros benefícios. Elas poderão ser utilizadas tanto pelas pessoas da área de negócios como para os engenheiros de software, facilitando assim o entendimento entre ambos, focando o sistema e os processos à um resultado único, facilitando reuso de conhecimento e retrabalho. Os engenheiros de software podem usar a estrutura das ontologias para definir seus requisitos, criar as classes e modelar o sistema através das ontologias criadas com auxilio dos modelos da metodologia CommonKADS proposta por Schreiber (2002), sendo assim, devemos nos preocupar também com a engenharia reversa, ou seja, quando o engenheiro por qualquer razão modificar a maneira como os processos serão desenvolvidos, mudando assim o mapa do conhecimento, a ontologia utilizada deverá ser atualizada para que também os analistas de negócios saibam das modificações realizadas. 
O sistema desenvolvido dessa forma, deverá representar em sua totalidade a abstração do negócio da empresa, de forma otimizada em relação a custos e tempo, assim como documentação, uso e reuso do conhecimento dos processos da empresa, sem falar no alinhamento do que foi pretendido, com o que efetivamente foi realizado. A figura 1 ilustra a proposta.

Figura 1: Proposta para Desenvolvimento de Sistemas de Conhecimento com uso da Metodologia CommonKADS e Ontologias.

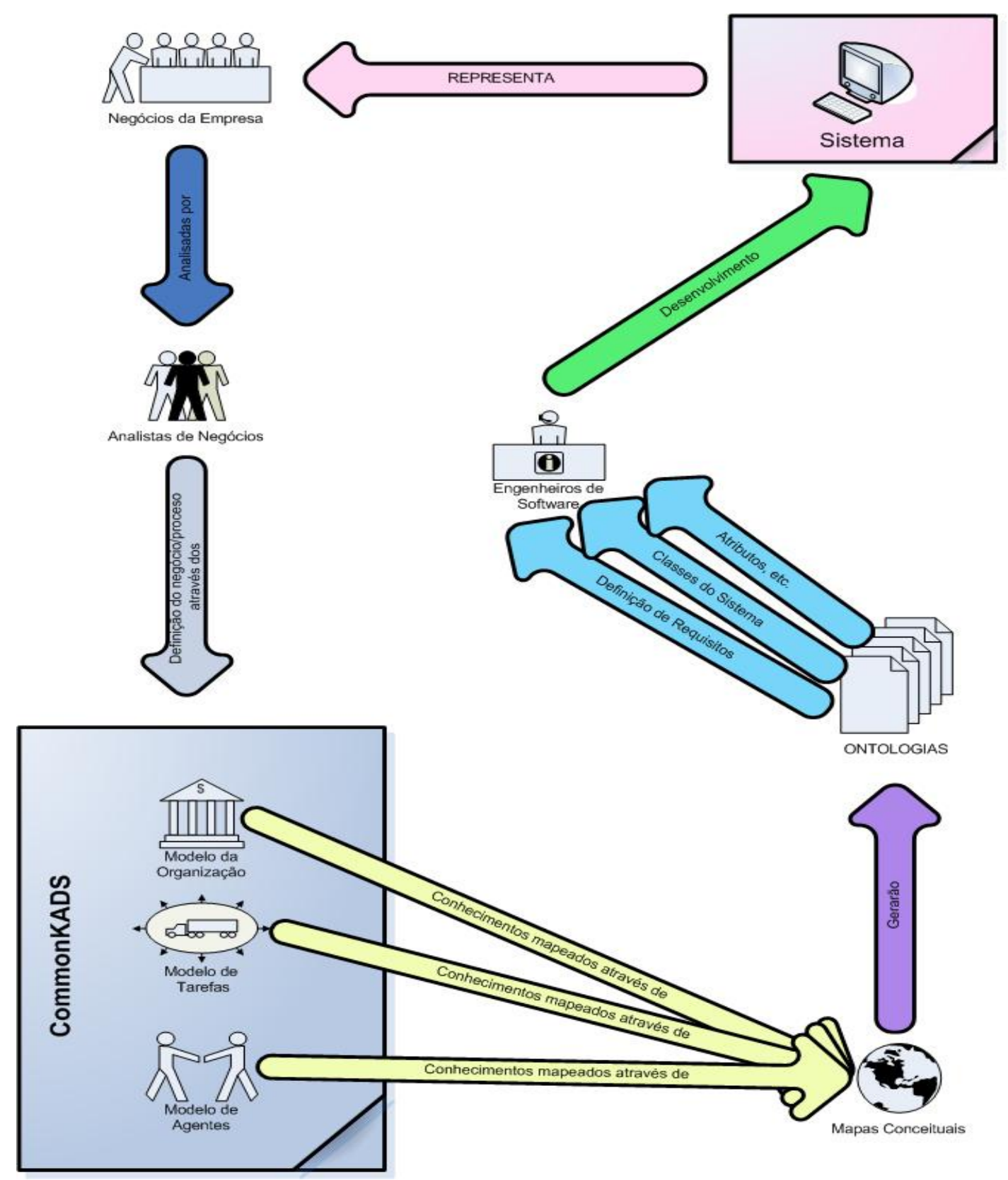

\section{Conclusões}


A aplicação proposta visa oferecer uma solução para o reuso de conhecimento e a criação de uma linguagem comum entre Analistas de Negócios e Engenheiros de Software, contribuindo diretamente para a facilitação das etapas que envolvem o desenvolvimento do sistema de conhecimento, assim como utilizar três dos modelos da Metologia CommonKADS para auxiliar no mapeamento do conhecimento e criação das ontologias.

Segundo o que foi exposto, é de fundamental importância que o conhecimento adquirido e utilizado pelos processos de negócios da empresa seja facilmente resgatado para facilitar o reuso deste conhecimento e assim como das informações, parâmetros e demais especificações referentes aos sistemas computacionais utilizados. Exatamente neste espaço seria desejável a criação de uma linguagem comum entre Analistas de Negócios e Engenheiros de Software, como elemento facilitador no entendimento e na documentação dos processos que compõe um sistema.

A perspectiva do uso de ontologias está se consolidando como uma das melhores alternativas para representar o conhecimento dos processos de negócios da empresa, sendo também um meio para facilitar operações de representação, alteração e análise deste conhecimento através do uso de editores de ontologias já disponíveis.

Para a consolidação de um avanço desta proposta, sugere-se a realização de uma implementação e aplicação experimental em uma organização, com a finalidade de validar os reais benefícios e avaliar as dificuldades que poderão ser encontradas para, desta forma, obter o feedback necessário ao seu aprimoramento.

\begin{abstract}
Currently the knowledge systems, as well as the other systems, are complex, including multiple knowledge areas, bringing and integrating all the company's oriented business processes, with the purpose to get benefits and competitiveness. However for system development, innumerable difficulties exist, as: agreement between oriented business analysts and software developers, difficulting the understanding of what it is to be done of what effectively it was done, rework between both parts to get the final result, opponent documentation, time, cost, requirements creation, etc. Therefore the present work considers to use the ontologies as a common language between oriented business analysts and software developers in order to eliminate misunderstandings, rework, facilitate reuse, among others benefits, and also to consider an alignment between ontologies and CommonKADS methodology (Schreiber, 2002), facilitating the system modeling.
\end{abstract}

Key-words: ontologies; business analysts; software engineer; commonkads; knowledge system.

\title{
Referências
}

ARENAS, Alvaro E., LAMBERT, Simon C., e MILES, Alistair J.. Engineering Knowledge-Intensive Tasks in Public Organisation. 2004.

AUSUBEL, D.P., NOVAK, J.D., HANESIAN, H. Psicologia Educacional. 2.ed. Rio de Janeiro: Interamericana, 1980.

DEVEDZIC, V. Ontologies: Borrowing from Software Patterns. Intelligence, Fall 1999. 
FREITAS, F. Ontologias e a Web Semântica In: Anais do XXIII Congresso da Sociedade Brasileira de Computação. 2001. Jornada de Mini-Cursos em Inteligência Artificial. Sociedade Brasileira de Computação, v.8, p. 1-52. Disponível em: http://www.inf.unisinos.br/ renata/cursos/topicosv/ontologias-ws.pdf. Acesso em: 15 de Maio de 2006.

GÓMEZ-PEREZ, A. Tutorial on Ontological Engineering. Internacional Joint Conference on Artificial IntelligenceIJCAI'1999. Estocolmo, Suécia. http://www.ontology.org/main/papers/madrid-tutorials.html. Acesso em: 15 de Maio de 2006.

GRUBER, T. R. Towards Principles for the Design of Ontologies Used for Knowledge Sharing. International Journal of Human and Computer Studies, 43(5/6): 907-928. $1995 . \quad$ Disponível em: http://citeseer.ist.psu.edu/gruber93toward.html. Acesso em:

10 de Maio de 2006.

GRUNINGER, M.; LEE, J. Ontology: Applications and Design. Communications of the ACM, vol. 45, no. 2, February 2002.

GUARINO, N. Formal Ontology and Information Systems. In: Proceedings of the First Int. Conference on Formal Ontology in Information Systems, Trento, Italy, June 1998. Disponível em: http://www.loa-cnr.it/Papers/FOIS98.pdf. Acesso em: 15 de Maio de 2006.

HOLSAPPle, C. H.; JOSHI, K. D. A Collaborative Approach to Ontology Design. Communications of the ACM, vol. 45, no. 2, February 2002.

cross ${ }^{\text {ref }}$

LENAT, D. B; GUHA, R. V. Building Large Knowledge Based Systems. Reading, Massachusetts: Addison Wesley 1990

MOREIRA, M. A., BUCHWEITS, B. Novas estratégias de ensino e aprendizagem: os mapas conceptuais e o vê epistemológico. Lisboa: Plátano, 1993.

PALlAZO, L. A. M. Aspectos da Modelagem de Sistemas de Informações Inteligentes. UFRS, Tese de Doutorado, 1996.

PROBST, G.; et al. Gestão do Conhecimento: os elementos construtivos do sucesso. Porto Alegre: Bookman, 2002.

PROTEGE. Disponível em: http://protege.stanford.edu/. Acesso em: 17 de Maio de 2006

SCHREIBER, G; AKKERMANS, H.; ANJEWIERDEN, A.; HOOG, R.; SHADBOLT, N.; DE VELDE, W. V.; and WIELINGA, B.. Knowledge Engineering and Management. The CommonKADS Methodology. MIT Press. Cambridge, Massachussets. 2002.

SILVA, L. F. da. Modelagem Conceitual como Ferramenta para o Desenvolvimento de Sistemas Computacionais. 1999. Disponível em: http://www.inf.puc-rio.br/ lyrene/\#publicacoes. Acesso em: 8 de Maio de 2006.

SOWA, J. F. Knowledge Representation - Logical, Philosophical and Computational Foundations. Brooks/Cole, USA, 2000 .

STEWART, Thomas. Capital intelectual: a nova vantagem competitiva das empresas. Rio de Janeiro: Campus, 1998.

SUTCLIFFE, A. G. Human-Computer Interface Design. Hampshire (London), Macmillan, 1995. 2a Ed. 326.

USCHOLD, M; GRUNINGER, M. Ontologies: Principles, Methods and Applications. The Knowledge Engineering Review, Vol. 11:2, pp 93-136, 1996.

cross'

\section{Dados dos autores:}

Nome completo: Erika Suzuki

Filiação institucional: Universidade Federal de Santa Catarina 
Departamento: Engenharia e Gestão do Conhecimento - EGC

Função ou cargo ocupado: estudante

Endereço completo: Protásio Alves, 7355 apto. 203 bloco 5, CEP: 91310-001, Porto Alegre - RS

Telefones para contato: (51) 91236603

e-mail: erika.suzuki@gmail.com

Nome completo: Vanessa Suzuki

Filiação institucional: Universidade Federal de Santa Catarina

Departamento: Engenharia e Gestão do Conhecimento - EGC

Função ou cargo ocupado: estudante

Endereço completo: Rua Dr. João Colin, 2008 apto. 101 bloco C, América, Joinville - SC,

CEP: 89204-000

Telefones para contato: (47) 34275386

e-mail:vanessa.suzuki@egc.ufsc.br

Nome completo: Aline França de Abreu

Filiação institucional: Universidade Federal de Santa Catarina

Departamento: Engenharia de Produção e Sistemas

Função ou cargo ocupado: professora

Endereço completo: UFSC - CTC - EPS Trindade, Florianópolis, SC, Brasil, CEP 88010-970

Telefones para contato: (48) 37217030

e-mail:aline@deps.ufsc.br

Nome completo: Wilton Souza

Filiação institucional: Universidade Federal de Santa Catarina

Departamento: Engenharia e Gestão do Conhecimento - EGC

Função ou cargo ocupado: estudante

Endereço completo: UFSC - CTC - EPS Trindade, Florianópolis, SC, Brasil, CEP 88010-970

Telefones para contato: (48) 88059438

e-mail:wsouza@inf.ufsc.br 